\title{
A Computational Multi-Criteria Optimization Approach to Controller Design for Physical Human-Robot Interaction
}

\author{
Yusuf Aydin, Member, IEEE, Ozan Tokatli, Member, IEEE, \\ Volkan Patoglu, Member, IEEE, and Cagatay Basdogan, Member, IEEE
}

\begin{abstract}
Physical human-robot interaction (pHRI) integrates the benefits of human operator and a collaborative robot in tasks involving physical interaction, with the aim of increasing the task performance. However, the design of interaction controllers that achieve safe and transparent operations is challenging, mainly due to the contradicting nature of these objectives. Knowing that attaining perfect transparency is practically unachievable, controllers that allow better compromise between these objectives are desirable. In this paper, we propose a multi-criteria optimization framework, which jointly optimizes the stability robustness and transparency of a closed-loop pHRI system for a given interaction controller. In particular, we propose a Pareto optimization framework that allows the designer to make informed decisions by thoroughly studying the trade-off between stability robustness and transparency. The proposed framework involves a search over the discretized controller parameter space to compute the Pareto front curve and a selection of controller parameters that yield maximum attainable transparency and stability robustness by studying this trade-off curve. The proposed framework not only leads to the design of an optimal controller, but also enables a fair comparison among different interaction controllers. In order to demonstrate the practical use of the proposed approach, integer and fractional order admittance controllers are studied as a case study and compared both analytically and experimentally. The experimental results validate the proposed design framework and show that the achievable transparency under fractional order admittance controller is higher than that of integer order one, when both controllers are designed to ensure the same level of stability robustness.
\end{abstract}

Index Terms-Multi-criteria optimization, interaction controllers, physical human-robot interaction (pHRI), fractional order control, transparency-stability robustness trade-off.

\section{INTRODUCTION}

$\mathbf{R}$ OBOTS are superior to humans at tasks that require precision, strength, and repetition, while the problem solving skills and adaptability of humans are unmatched thanks to their cognitive abilities. Physical human-robot interaction (pHRI) integrates the benefits of human and robot in tasks that involve physical interaction [1]. From assembly tasks to furniture relocation tasks in home/office setting [2], from industrial applications [3], surgery [4], rehabilitation [5] to mission critical tasks such as manipulation in hazardous

Y. Aydin and C. Basdogan are with the College of Engineering, Koc University, Istanbul, 34450 Turkey. E-mail: yaydin@ku.edu.tr, cbasdogan@ku.edu.tr.

O. Tokatli is with RACE UKAEA, Abingdon, OX14 3DB, UK. E-mail: ozan.tokatli@ukaea.uk.

V. Patoglu is with Faculty of Engineering and Natural Sciences, Sabanci University, Istanbul, 34956, Turkey. E-mail: vpatoglu@ sabanciuniv.edu.

Manuscript received October 31, 2019; revised March 22, 2020. environments [6], collaboration of humans and robots brings high performance solutions to complex problems (please refer to extensive reviews in [7]-[10]). In such scenarios, the stability of the coupled human-robot dyad must be ensured and the controller should be sufficiently robust to the changes in both human and environment dynamics for safe operation, while the dyad targets to achieve a high task performance, which typically requires the robot to be transparent to the human operator. However, the contradicting nature of robust stability and high transparency requirements creates challenges during the design of interaction controllers. In this study, we propose a computational multi-criteria optimization approach to design interaction controllers for pHRI to address the tradeoff between stability robustness and transparency.

\section{A. Related Work}

The controller design for pHRI systems must pay utmost attention to the coupled stability of the human-in-the-loop system. However, due to the complexity introduced by the presence of human operator and possible contact interactions with uncertain environments, stability characteristics of pHRI systems cannot be analysed easily.

Different approaches have been used to study coupled stability of interaction. Model based approaches [11]-[16] rely on a simplified model of human dynamics and environment that capture most essential characteristics of these systems in terms of stability. In particular, given that the stiffness of the human arm and the environment have a direct effect on coupled stability [11], [14], [15], most of these models focus on capturing this dominant aspect. In the absence of human and environment models, the coupled stability of pHRI systems can be investigated using the frequency domain passivity framework [17]-[19]. In this approach, the human operator and the environment are assumed to act as passive elements that do not inject energy to the closedloop system; hence, do not tend to destabilize the closedloop system. While frequency domain passivity can guarantee the stability of the closed-loop system for a broad range of human/environment models, the resulting controllers perform conservatively [18]-[21], leading to a less transparent performance. Time domain passivity framework [20] aims to relax the conservativeness of the frequency domain passivity framework by continually estimating the exchanged energy between robot and human and/or environment through sensor measurements and dissipating any excess energy as needed. 
Satisfying passivity throughout the interaction ensures that the robot behaviour is never active (i.e. energy is not generated), so that stability can be guaranteed. However, nonpassive systems are not necessarily unstable [22]. This is due to the fact that passivity only considers the phase bounds on the uncertainty and assumes that the magnitude of this uncertainty can be arbitrarily large. In practice, many systems are not exposed to such large changes or uncertainties. The conservativeness of the passivity framework can be relaxed by taking advantage of partial knowledge (e.g., magnitude bounds) on the models of human and/or environment. For instance, even though the dynamics of human arm changes over time, human arm impedance is known to vary in a relatively limited range [23], [24]. Along these lines, Buerger and Hogan [25] proposed a complementary stability approach to design interaction controllers that can maintain robust stability for bounded ranges of impedances and coupled stability is ensured without the need for passivity. Similarly, in [26], it is shown that passivity constraints can be relaxed when the bounds on environmental/human impedances are known.

The trade-off between robust stability and transparency brings a challenge in pHRI tasks that require high transparency [27]. This trade-off, as well as the factors affecting the transparency, have been investigated in detail [28]-[34]. As an indicator of the achievable transparency, Z-width, the bounds of the dynamic range of achievable impedances, was proposed by Colgate and Brown [35] and methods to improve the Z-width were studied in [36], [37]. Yet, it is well known that attaining perfect transparency is practically infeasible [27]. Hence, while keeping coupled stability intact, a controller allowing for an optimal compromise between transparency and robustness is desirable for pHRI studies [34].

Recently, we proposed the use of fractional order admittance controllers (FOAC) for pHRI, compared their performance with conventional integer order admittance controllers (IOAC) and provided evidence that FOAC can offer better stability robustness while displaying higher transparency than IOAC [38]. In particular, we introduced the concept of impedance matching to enable comparisons between FOAC and IOAC at a given frequency and analytically showed that both stability robustness and transparency performance can be improved under FOAC [15].

Earlier studies have already shown that a compromise between stability robustness and transparency is possible by manipulating the parameters of an interaction controller for pHRI [15], [25], [38]. Nevertheless, analytical methods have proven to be prohibitive for the design of an optimal interaction controller for $\mathrm{pHRI}$, due to the non-trivial interaction between the control parameters and the dynamics of the coupled system. On the other hand, computational approaches have been quite promising. Computational optimization approaches have been proposed in [25], [39] to design controllers with improved performance. While stability and transparency have been considered in these studies, both studies address single objective optimization problems. In particular, in [25] stability is imposed as a constraint, while in [39] multiple criteria are aggregated into a single cost function through predetermined weights.

\section{B. Contributions}

In this study, we propose a computational multi-criteria optimization approach to design linear time-invariant (LTI) interaction (i.e., admittance and impedance) controllers for pHRI tasks. To our knowledge, no such multi-criteria optimization framework has been proposed to simultaneously optimize interaction controllers for multiple objectives, such as stability robustness and transparency.

Since the dynamics of interaction among human, robot and the environment are highly complex, and rendering the development of an analytical approach is prohibitive, a computational approach is proposed. Earlier computational studies for optimizing the human-robot interaction have focused on determination of joint manipulation trajectory [40], [41], improvement of task performance [42]-[44], collaborative task planning and scheduling [45], [46], but not on the trade-off between robust stability and transparency.

Buerger and Hogan [25], and Labrecque and Gosselin [39] have proposed single criterion optimization of interaction controllers. In these approaches, the original multi-objective problem is formulated as a single criterion optimization problem, either by considering stability (robustness) as a constraint and/or by scalarizing the cost functions using predetermined weights to define a single aggregate objective function. The drawback of these approaches is that the preferences between objectives need to be assigned a priori, before having a complete knowledge on the trade-offs involved.

On the other hand, the proposed Pareto optimization approach computes all possible non-dominated solutions that form the Pareto front curve and allows the designer to make informed decisions on optimal interaction controllers. Unlike one-shot scalarization-based optimization methods, Pareto methods fully characterize the trade-off among objectives by providing all optimal solutions for all possible preferences of the designer. This allows an optimal solution to be selected after the trade-off is thoroughly studied, possibly by considering new criteria that have not been considered during optimization. Furthermore, Pareto methods allow the designer to choose alternative optimal solutions under different conditions.

In addition to multi-criteria optimal design of interaction controllers, the use of Pareto optimization approach enables fair comparisons among different controller structures, as comparison can be performed based on the best possible performance of controllers for all possible user preferences. We demonstrate such a comparison as a case study that compares FOAC and IOAC and by experimentally verifying that achievable transparency under FOAC is higher than that of IOAC, when same level of stability robustness is required.

The rest of this paper is organized as follows: Section II introduces our computational interaction controller design approach. Section III presents running examples for which the interaction control architectures, and transparency and stability robustness metrics are introduced. Pareto optimization is presented in Section II-C. Human subject experiments conducted to assess the transparency under optimal FOAC and IOAC are reported in Section IV. Discussions and conclusions are provided in Section $\mathrm{V}$. 


\section{Multi-Criteria Design Optimization ApPROACH}

\section{A. Designing Controllers for Multiple Objectives}

Stability robustness and transparency performance analyses of interaction controllers are challenging, as the standard tools used for servo control cannot be applied to controller design. Two fundamental differences exist for interaction control systems as noted in [25]: (i) the closed-loop stability and performance cannot be predicted and characterized using the openloop transfer function alone, and (ii) the controller parameters do not directly affect the stability and performance of the coupled system. Consequently, there exists no straightforward way to investigate the stability and performance of such systems analytically, while computational approaches have been shown to provide promising results [19], [25], [47].

Buerger and Hogan [25] used a computational loop-shaping approach to optimize an interaction controller for maximum performance while satisfying a stability constraint. In their approach, first, the stability of the closed-loop system is evaluated in the controller parameter space that covers all possible combinations of controller parameters and then, the controller parameters that yield the maximum performance among the stable solutions are selected.

We advocate Pareto methods for multi-criteria optimization of interaction controllers. Given a multi-criteria optimization problem, all non-dominated solutions constitute the Pareto front [48]-[50]. Every Pareto optimization approach consists of three main steps: i) selection and evaluation of cost functions, ii) computation of the Pareto front, and iii) selection of an optimal solution among all non-dominated solutions. Below we provide an outline of the proposed approach.

\section{B. Objective Functions for Interaction Controllers}

Depending on the task, the performance of a pHRI application can be assessed using various criteria. In this study, we focus on the two fundamental ones which are known to possess an inherent trade-off: stability robustness and transparency.

The first objective function is chosen as the stability robustness of the closed-loop system. The stability is an integral part of robotic applications, especially in pHRI domain, where it implies inherent safety of the operator. For pHRI applications, a degree of robustness in the stability of the closed-loop system needs to be guaranteed, since elements of the closed-loop transfer function, e.g. human and environment, are subject to change during the task execution. Under these circumstances, a controller that can provide closed-loop stability over a desired range of parameter variations is desirable.

The second objective is chosen as the transparency of the pHRI task. The impedance that a human feels during interactions with an environment has critical importance in many pHRI tasks. For instance, in a robotic assisted surgery, the control architecture for the interaction is expected to ensure that the impedance of the soft tissue is reflected to the surgeon so that she/he can have a realistic understanding of the operation, without being shadowed by the dynamics of the robot. Alternatively, in a different task, an operator may wish to move the robot in free space, where she/he does not desire to feel any parasitic dynamics due to the robot.

\section{Pareto Optimization}

Assuming an LTI model of the robot is available and an LTI controller structure to regulate the interaction between the robot and the human is selected, the following steps are proposed to optimize the controller parameters for a robust and transparent design.

Step 1) A feasible range of values for each parameter of the interaction controller is chosen (and discretized). Feasible controller parameters are computed as design variables by considering these range of values for each parameter.

Step 2) A metric for transparency is defined for the design variables in Step 1.

Step 3) A metric for stability robustness is defined for the design variables in Step 1.

Step 4) Using a Pareto optimization approach, the Pareto front curve is constructed by considering the transparency and robustness objectives.

While many different approaches have been proposed in the literature to compute the Pareto front, we utilize the weightedsum approach with weight scanning [49] in this study, as this approach is easy to implement and present.

\section{Selection of Optimal Controller}

The resulting Pareto front represents all non-dominated solutions for the given design problem and these solutions reflect optimal solutions for different preferences among the selected metrics. Once the Pareto front is computed, the designer is expected to study these solutions to get an insight of the underlying trade-offs and make an informed decision to finalize the controller design by selecting an optimal solution from the Pareto set. Constraints or new criteria that have not been considered during the optimization can be introduced at this stage. Pareto methods allow the designer to choose alternative optimal solutions under different conditions.

The Pareto optimization approach is rewarding as it not only leads to design of optimal interaction controllers but also enables fair comparisons among various interaction controllers, possibly with different underlying types or structures. Given that the Pareto front for each controller provides the best performance of that controller for all possible preferences of the designer, fair comparisons become possible by considering the Pareto front of each controller type/structure. Hence, for any given designer preference, the best possible performances of each controller is compared to the best possible performances of other controllers.

\section{Case Study: Interaction Controller Design}

This section presents a case study that follows the Pareto optimization approach introduced in Section II. In particular, a family of admittance controllers are studied in terms of stability robustness and transparency, and optimal controller parameters are determined from their Pareto solutions. 


\section{A. pHRI Tasks}

Three different pHRI scenarios are considered to demonstrate the proposed computational optimization framework in designing an interaction controller.

S1 Only the human and robot physically interact. Ideally, human operator desires not to feel the robot dynamics while moving the robot in free space.

S2 The human operator continually interacts with a nominal environment using the robot. Note that the dynamics of the environment does not change during the execution of the pHRI task (in other words, an LTI model for the environment is assumed).

S3 The human operator interacts with an environment using the robot, while the dynamics of the environment changes.

S1 involves no interaction with environment, so this scenario is considered as a baseline for S2 and S3. In S2, the contact with environment involves a linear spring. In S3, the dynamics of the environment is changed by considering two springs in parallel, where one of the springs is engaged only during a portion of the interaction. Along these lines, S3 involves switching dynamics resulting in a nonlinear interaction.

\section{B. The Closed-Loop System}

Figure 1 depicts the admittance control architecture used in our pHRI studies [15], [38], where the human, robot, and the environment physically interact with each other. The transfer function of the closed-loop system is given by

$$
T(s)=\frac{V(s)}{F_{\text {int }}(s)}=\frac{G(s) Y(s)}{1+G(s) Y(s) H(s) Z_{\mathrm{eq}}(s)}
$$

where $G(s)$ is the LTI model of robot, $V(s)$ is its measured (actual) end-effector velocity, $Y(s)$ is the admittance controller, and $H(s)$ models a filter introduced to attenuate the noise in force measurements. Human and environment are assumed to be coupled, and their equivalent impedance is set to $Z_{\mathrm{eq}}=Z_{h}+Z_{e}$, where $Z_{h}$ and $Z_{e}$ represent linearized human and environment impedances, respectively. The equivalent impedance used in our study is $Z_{\mathrm{eq}}(s)=\frac{m_{\mathrm{eq}} s^{2}+b_{\mathrm{eq}} s+k_{\mathrm{eq}}}{s}$, where equivalent stiffness, damping, and mass elements can be defined as $k_{\mathrm{eq}}=k_{h}+k_{e}, b_{\mathrm{eq}}=b_{h}+b_{e}$, and $m_{\mathrm{eq}}=m_{h}+m_{e}$, respectively.

FOAC used in this study has the following form

$$
Y(s)=\frac{1}{Z_{\mathrm{FOAC}}}=\frac{1}{m_{F} s^{\alpha}+b_{F}}
$$

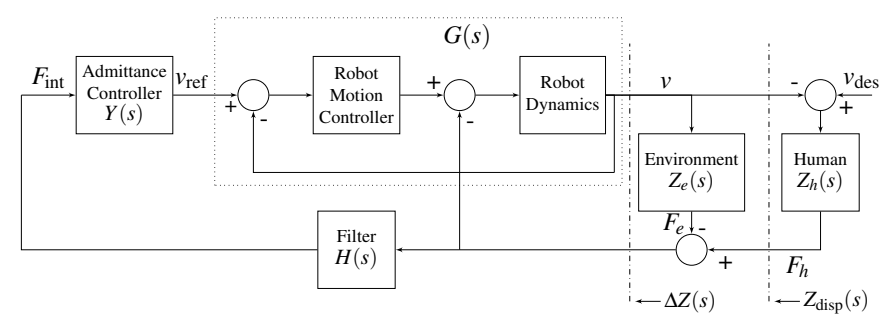

Fig. 1. Control architecture of the pHRI system where $\alpha$ corresponds to the order of the fractional integrator (i.e., the integration order), while $m_{F}$ and $b_{F}$ are the admittance controller parameters. In this study, the integration order is kept in the range of $0<\alpha \leq 1$. In the limit case when $\alpha=1$, the FOAC becomes equivalent to IOAC; therefore, the admittance controller becomes $Y(s)=1 / Z_{\mathrm{IOAC}}=1 /\left(m_{F} s+b_{F}\right)$.

As the unit of $m_{F}$ is $\mathrm{kgs}^{\alpha-1}$, the physical interpretation of this parameter changes depending on the integration order $\alpha$, whereas the unit of $b_{F}$ is always $\mathrm{Ns} / \mathrm{m}$. The effective mass and damping provided by FOAC are $m_{F} \omega^{\alpha-1} \sin \left(\frac{\alpha \pi}{2}\right) \mathrm{kg}$ and $b_{F}+m_{F} \omega^{\alpha} \cos \left(\frac{\alpha \pi}{2}\right) \mathrm{Ns} / \mathrm{m}$, respectively, where $\omega$ represents the frequency. Thorough analyses of the effective impedance of the fractional order interaction controllers can be found in [15], [38], [51], [52].

Knowing that controller parameters have different physical interpretations and the structure of an admittance controller is different for each integration order $\alpha$, a fair comparison among controllers of different orders is challenging. Therefore, being able to thoroughly investigate and rigorously compare the trade-off between stability robustness and transparency under various integration orders by using the optimization framework proposed in this study is a rewarding practice.

\section{Bounds on Equivalent Impedance $\left(Z_{e q}\right)$}

In this section, we determine the bounds on equivalent impedance $Z_{\mathrm{eq}}$ for each pHRI scenario.

For the first scenario (S1), only the bounds of the human arm impedance is considered, since $Z_{e}(s)=0$. Lower and upper bounds for the human arm stiffness are taken as $k_{h}=0$ (when human is not in contact with the end-effector of the robot) and $k_{h}=600 \mathrm{~N} / \mathrm{m}$ (based on [23], [24]), respectively. Moreover, the lower and upper bounds for the mass parameter are set to $m_{h}=0$ (when human releases her/his contact) and $m_{h}=5 \mathrm{~kg}$, respectively. The range of damping for the human arm $b_{h}$ is taken as 0 to $41 \mathrm{Ns} / \mathrm{m}$, as proposed in [23], [24].

For the second scenario (S2), the contact interaction with a spring-like environment having a stiffness of $k_{e}$ is considered. Therefore, the damping, and the mass of the equivalent impedance are kept the same as damping and mass of human arm as in S1: $b_{\text {eq }}=b_{h}$ and $m_{\text {eq }}=m_{h}$. The environment is assumed to have a nominal stiffness of $k_{e}=610 \mathrm{~N} / \mathrm{m}$, thus the range for the equivalent stiffness is set to $610 \mathrm{~N} / \mathrm{m} \leq k_{\mathrm{eq}} \leq$ $1210 \mathrm{~N} / \mathrm{m}$.

Similarly, for the third scenario (S3), the environment stiffness varies between $610 \mathrm{~N} / \mathrm{m} \leq k_{e} \leq 1010 \mathrm{~N} / \mathrm{m}$, thus, the range for the equivalent stiffness is set to $610 \mathrm{~N} / \mathrm{m} \leq k_{\text {eq }} \leq$ $1610 \mathrm{~N} / \mathrm{m}$.

\section{Computation of the Pareto Front}

\section{Step 1 - Feasible Controller Parameters}

For a given integration order $\alpha, m_{F} \in[0.2,100] \mathrm{kgs}^{\alpha-1}$ and $b_{F} \in[0.001,500] \mathrm{Ns} / \mathrm{m}$ are taken as the feasible range of the admittance controller parameters and these ranges are discretized with equally spaced increments of $0.1 \mathrm{kgs}^{\alpha-1}$, and $1 \mathrm{Ns} / \mathrm{m}$, respectively. 


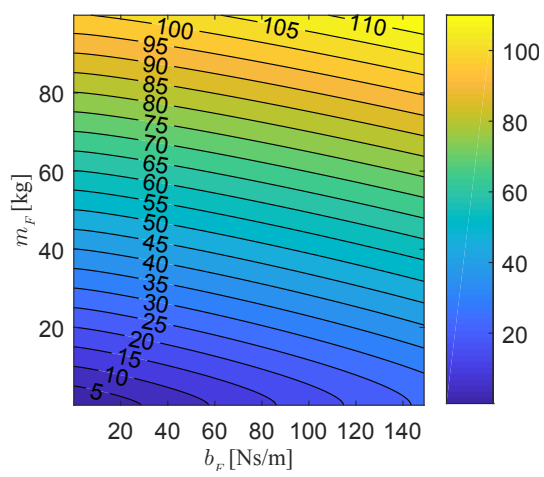

(a) $\alpha=1$

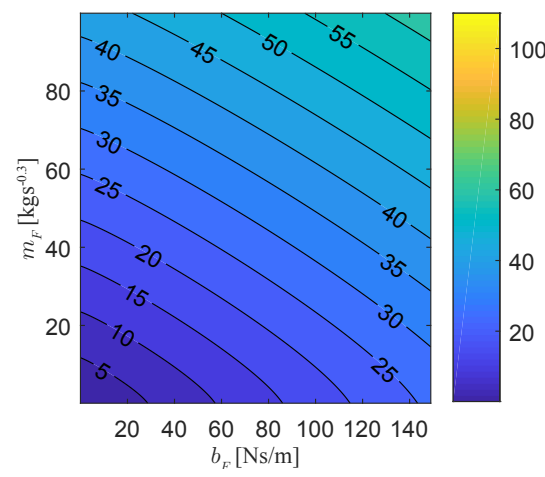

(b) $\alpha=0.7$

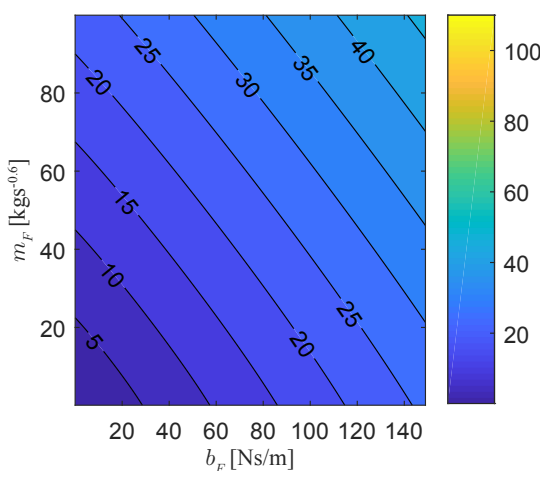

(c) $\alpha=0.4$

Fig. 2. Transparency maps illustrating the parasitic impedance of the controller in Figure 1 under (a) IOAC $\alpha=1$, (b) FOAC $\alpha=0.7$, and (c) FOAC $\alpha=0.4$.

\section{Step 2 - Transparency Cost Function}

The closed-loop impedance displayed to the human operator $Z_{\mathrm{disp}}(s)$ according to Figure 1 can be computed as

$$
Z_{\text {disp }}(s)=\frac{F_{h}(s)}{V(s)}=\frac{1+G(s) Y(s) H(s) Z_{e}(s)}{G(s) Y(s) H(s)}
$$

The parasitic impedance $\Delta Z(s)$ is defined as the difference between the desired impedance $Z_{\mathrm{des}}(s)$ and the impedance reflected to human $Z_{\mathrm{disp}}(s)$ :

$$
\Delta Z(s) \triangleq Z_{\text {des }}(s)-Z_{\text {disp }}(s)
$$

If the parasitic impedance $\Delta Z(s)$ is small, then the transparency of the overall system is high. In most pHRI applications, the desired impedance is equal to the environment impedance, $Z_{\mathrm{des}}(s)=Z_{e}(s)$. In this condition, considering (3) and (4), the magnitude of parasitic impedance in frequency domain can be determined as follows:

$$
|\Delta Z(j \omega)|=1 /|G(j \omega) Y(j \omega) H(j \omega)|
$$

Clearly, maximizing $|G Y H|$ minimizes $|\Delta Z|$, which in turn maximizes the transparency. Furthermore, in terms of the controller, we can deduce that maximizing the magnitude of $Y(j \omega)$ is required to improve the transparency. According to $Y(j \omega)$, lower values of $m_{F}$ and $b_{F}$ result in higher transparency. At low frequencies, the effect of $b_{F}$ is more dominant on the parasitic impedance. Therefore, lower values of $b_{F}$ are more desirable for higher transparency at low frequencies. Moreover, effects of $\alpha$ and $m_{F}$ become increasingly more dominant at higher frequencies.

While inspection of the magnitude of $Y(j \omega)$ provides insight about how controller parameters affect the transparency, the parasitic impedance function should be studied for more conclusive results, since the dynamics of robot itself also contributes to the parasitic impedance. In particular, the fact that the contributions of controller and robot on parasitic impedance are complex numbers and change as functions of frequency calls for a quantitative cost metric for the parasitic impedance. Such a metric was defined in [25] and computed over a discrete range of frequencies. Adopting this metric, the transparency cost function is defined as

$$
C=\sum_{\omega_{L}}^{\omega_{U}} W(\omega) \log |\Delta Z(j \omega)|
$$

where $W(\omega)$ is a weighting function defined to adjust contributions at each frequency, and $\omega_{L}$ and $\omega_{U}$ represent the lower and upper bounds of the frequency range of interest, respectively.

In this study, a logarithmically spaced frequency ranging from $0.01 \mathrm{~Hz}$ to $30 \mathrm{~Hz}$ is chosen for the discretization. Since the frequency range of human voluntary movements is around $2 \mathrm{~Hz}$ [53], achieving higher transparency is more desirable at lower frequencies. Along these lines, a fifth order Butterworth filter with a cut-off frequency of $5 \mathrm{~Hz}$ is used as the weighting function $W(\omega)$ to boost the effect of low frequency content on the parasitic impedance and reduce the contribution of higher frequencies. Using (6), parasitic impedance for each set of controller parameters is evaluated, and the resulting transparency maps illustrating the parasitic impedance are constructed as in Figure 2. As expected, lowering $m_{F}$, and $b_{F}$ reduce the parasitic impedance, leading to higher transparency.

\section{Step 3 - Stability Robustness Cost Function}

We utilize the vector margin, defined as the inverse of the maximum magnitude of loop sensitivity function $S(s)$, as the metric of the stability robustness. For the system in Figure 1, the sensitivity transfer function is defined as $S(j \omega)=1 /(1+$ $L(j \omega))$, where $L(j \omega)=G(j \omega) Y(j \omega) H(j \omega) Z_{\text {eq }}(j \omega)$ denotes the loop transfer function. Then, the vector margin used as the cost function to quantify stability robustness, is defined as

$$
\rho=\frac{1}{\max (|S(j \omega)|)}
$$

where $\max (|S(j \omega)|)$ represents the maximum magnitude of the loop sensitivity function. From this equation, one can note that the larger the loop gain, the more robust the system.

Three sets of robustness maps for $\alpha \in\{1,0.7,0.4\}$ are generated as presented at different rows of Figure 3. Note that, these robustness maps are only constructed for the stable sets of controller parameters for the first pHRI scenario (S1), as this case is treated as a baseline for other scenarios ( $\mathrm{S} 2$ and S3) that involve interactions with an environment.

To generate worst-case stability robustness maps for each integration order $\alpha$, four different robustness margins are calculated for each stable parameter set: one for each combination of extreme values of equivalent mass $m_{\mathrm{eq}}$ and damping $b_{\mathrm{eq}}$. For any given controller parameter set, the minimum of the 


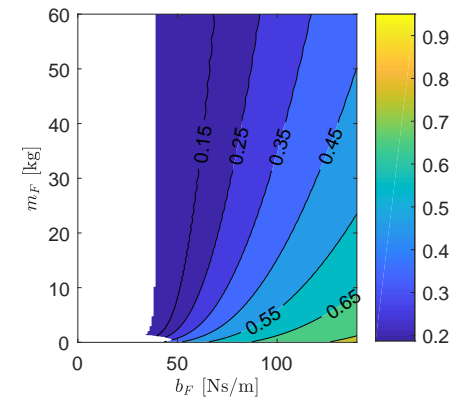

(a) $\alpha=1, m_{\mathrm{eq}}=0 \mathrm{~kg}$, $b_{\text {eq }}=0 \mathrm{Ns} / \mathrm{m}$

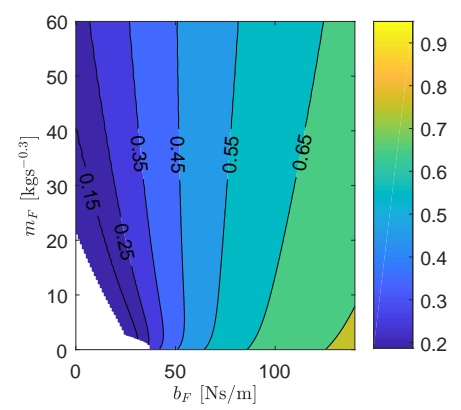

(e) $\alpha=0.7, m_{\mathrm{eq}}=0 \mathrm{~kg}$, $b_{\mathrm{eq}}=0 \mathrm{Ns} / \mathrm{m}$

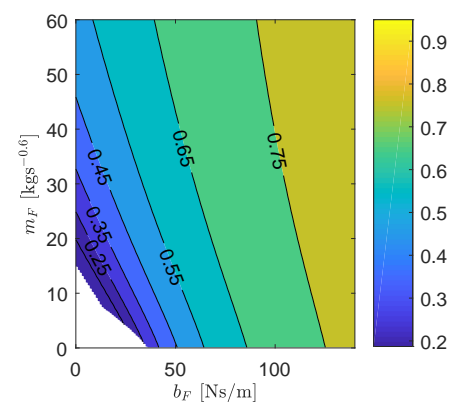

(i) $\alpha=0.4, m_{\mathrm{eq}}=0 \mathrm{~kg}$, $b_{\mathrm{eq}}=0 \mathrm{Ns} / \mathrm{m}$

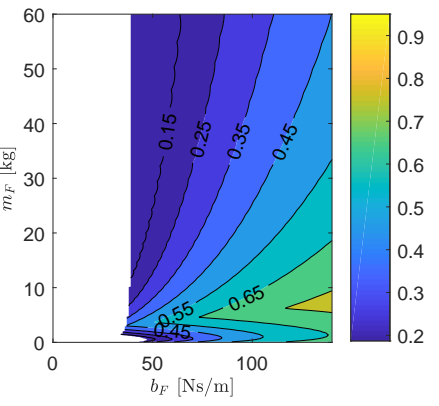

(b) $\alpha=1, m_{\mathrm{eq}}=5 \mathrm{~kg}$, $b_{\text {eq }}=0 \mathrm{Ns} / \mathrm{m}$

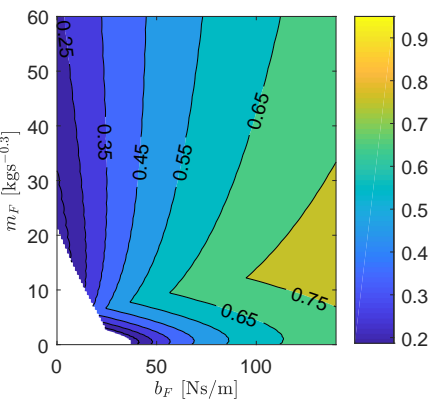

(f) $\alpha=0.7, m_{\mathrm{eq}}=5 \mathrm{~kg}$, $b_{\text {eq }}=0 \mathrm{Ns} / \mathrm{m}$

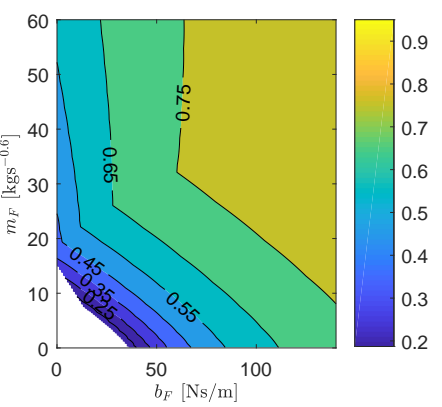

(j) $\alpha=0.4, m_{\mathrm{eq}}=5 \mathrm{~kg}$, $b_{\text {eq }}=0 \mathrm{Ns} / \mathrm{m}$

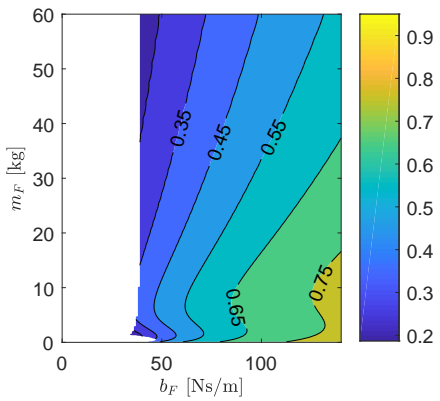

(c) $\alpha=1, m_{\mathrm{eq}}=0 \mathrm{~kg}$ $b_{\mathrm{eq}}=41 \mathrm{Ns} / \mathrm{m}$

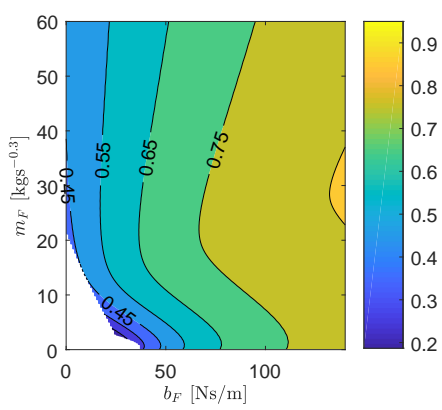

(g) $\alpha=0.7, m_{\mathrm{eq}}=0 \mathrm{~kg}$, $b_{\mathrm{eq}}=41 \mathrm{Ns} / \mathrm{m}$

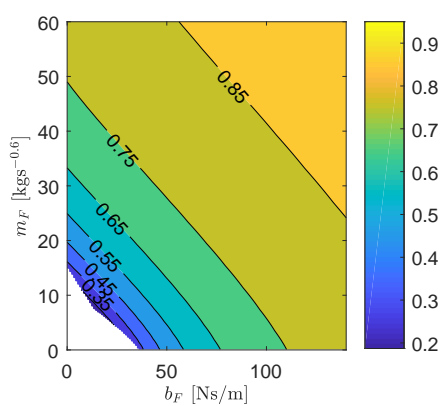

(k) $\alpha=0.4, m_{\mathrm{eq}}=0 \mathrm{~kg}$,

$b_{\text {eq }}=41 \mathrm{Ns} / \mathrm{m}$

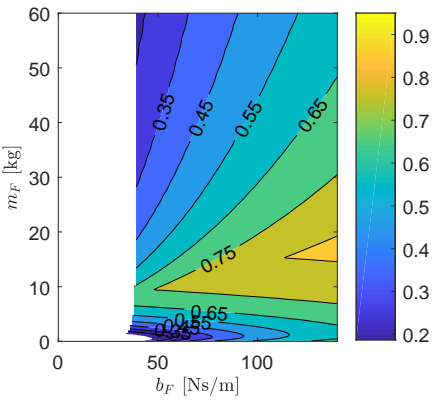

(d) $\alpha=1, m_{\mathrm{eq}}=5 \mathrm{~kg}$, $b_{\text {eq }}=41 \mathrm{Ns} / \mathrm{m}$

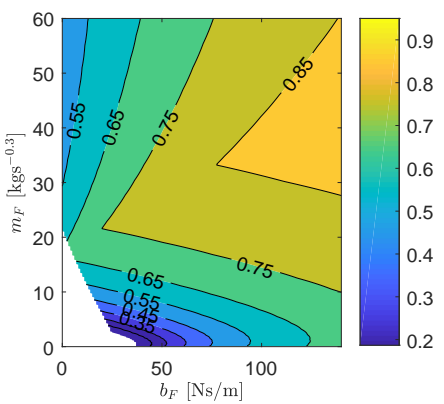

(h) $\alpha=0.7, m_{\mathrm{eq}}=5 \mathrm{~kg}$, $b_{\text {eq }}=41 \mathrm{Ns} / \mathrm{m}$

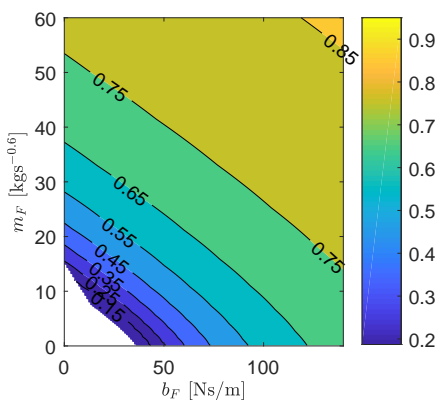

(l) $\alpha=0.4, m_{\mathrm{eq}}=5 \mathrm{~kg}$, $b_{\text {eq }}=41 \mathrm{Ns} / \mathrm{m}$

Fig. 3. Stability robustness maps of of the controller in Figure 1 for $k_{\mathrm{eq}}=600 \mathrm{~N} / \mathrm{m}$ under IOAC $\alpha=1$, FOAC $\alpha=0.7$, and FOAC $\alpha=0.4$.

robustness margins calculated for these four combinations of extreme values of $m_{\mathrm{eq}}$ and $b_{\mathrm{eq}}$ is taken as the conservative robustness margin, so that the worst-case uncertainties in $m_{\mathrm{eq}}$ and $b_{\text {eq }}$ are accounted for in the conservative robustness maps presented in Figure 4.

In Figure 4, the relation between the stability robustness margin and equivalent stiffness is depicted. These plots can be utilized to determine how much increase in equivalent stiffness can be tolerated in scenarios S2 and S3. In particular, it can be deduced that a maximum equivalent stiffness increase of $610 \mathrm{~N} / \mathrm{m}$ and $1010 \mathrm{~N} / \mathrm{m}$ can be tolerated for S2 and S3, respectively; that is, the maximum expected equivalent stiffness can be set to $k_{\mathrm{eq}}=1210 \mathrm{~N} / \mathrm{m}$ and $k_{\mathrm{eq}}=1610 \mathrm{~N} / \mathrm{m}$ for S2 and S3, respectively, without sacrificing stability.

The stability boundaries for $k_{\mathrm{eq}}=1210 \mathrm{~N} / \mathrm{m}$ and $k_{\mathrm{eq}}=$ $1610 \mathrm{~N} / \mathrm{m}$ are computed by employing the principles in [15] and superimposed on top of the stability robustness maps in Figure 4. One can observe from Figure 4a that among the controller parameter sets laying on the stability boundary for $k_{\text {eq }}=1210 \mathrm{~N} / \mathrm{m}\left(\right.$ resp. $\left.k_{\text {eq }}=1610 \mathrm{~N} / \mathrm{m}\right)$ under IOAC, the most robust one possesses a stability robustness of $\rho=0.42$ (resp. $\rho=0.55$ ). In other words, any controller parameter set of IOAC that results in $\rho=0.42$ (resp. $\rho=0.55$ ) ensures the coupled stability for S2 (resp. S3), under the impedance bounds defined for the human and the environment.

Any controller parameter set of FOAC ( $\alpha=0.7$ and 0.4 as presented in Figures $4 \mathrm{~b}$ and $4 \mathrm{c})$ that results in the same robustness margin of $\rho=0.42(\rho=0.55)$ also ensures coupled stability for S2 (resp. S3), as equivalent stiffness of $k_{\text {eq }}=1210 \mathrm{~N} / \mathrm{m}\left(\right.$ resp. $\left.k_{\text {eq }}=1610 \mathrm{~N} / \mathrm{m}\right)$ can be tolerated. Note that the controller parameters for FOAC having the stability robustness margin of $\rho=0.42(\rho=0.55)$ can tolerate even larger equivalent stiffness values as they lay further away from the stability boundary.

\section{Step 4 - Pareto Optimization}

We aim to minimize the transparency cost function $C$, while maximizing the value of the stability robustness margin $\rho$. To eliminate the scale difference between the two metrics, we first normalize each one with their maximum value, where 


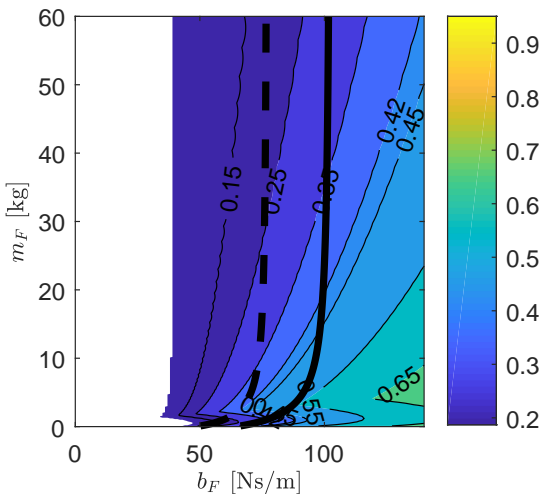

(a) $\alpha=1$

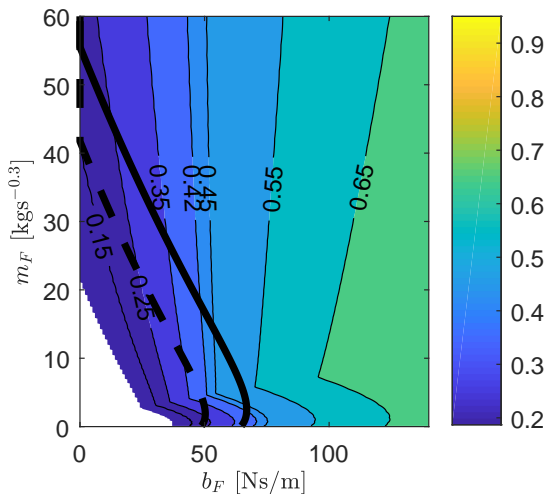

(b) $\alpha=0.7$

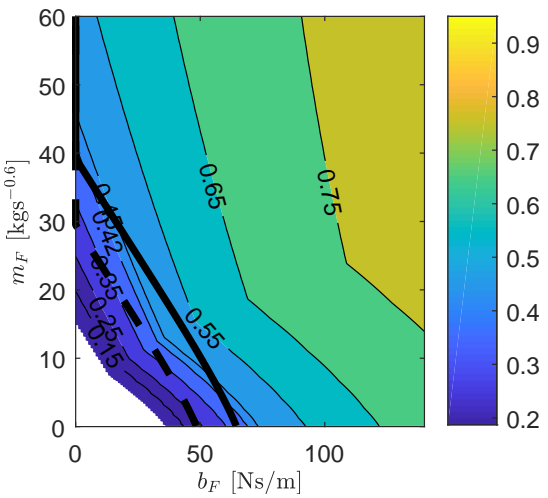

(c) $\alpha=0.4$

Fig. 4. Conservative stability robustness maps of the controller for $k_{\mathrm{eq}}=600 \mathrm{~N} / \mathrm{m}$ under (a) IOAC $\alpha=1$, (b) FOAC $\alpha=0.7$, and (c) FOAC $\alpha=0.4$. Solid and dashed thickened black curves show the stability boundaries for $k_{\mathrm{eq}}=1210 \mathrm{~N} / \mathrm{m}$ and $k_{\mathrm{eq}}=1610 \mathrm{~N} / \mathrm{m}$, respectively.

the maximum values are acquired from the transparency maps (Figure 2) and the stability robustness maps (Figure 4). Then, we form the final objective function as their convex combination according to

$$
J=w C_{n}+(1-w)\left(-\rho_{n}\right)
$$

where $w \in[0,1]$ represents the weights, while $C_{n}$ and $\rho_{n}$ denote the normalized values of transparency cost function and stability robustness margin, respectively. Each value of the weight parameter $w$ results a different optimization problem, corresponding to various prioritization of the cost functions. If the problem is solved for all possible weights $w \in[0,1]$, then resulting set of optimal solutions yield the Pareto front for the multi-criteria optimization problem.

During our implementation, the weight parameter $w$ is scanned with equally spaced increments of 0.001 to construct the Pareto front curve. Figure 5 presents the Pareto front curve for the integration order $\alpha=1$. A close inspection of Figure 5 reveals the trade-off between the selected cost functions. The maximum robustness margin is achieved when the parasitic impedance is at its maximum value, resulting in a poor transparency performance. On the contrary, the parasitic impedance attains its minimum, yielding to the highest transparency, when the robustness margin is at its minimum, leading to the lowest stability robustness.

Prioritization of objectives without having a thorough understanding on the trade-off characteristics may lead to conservative designs, whereas Pareto front curve characterizes the trade-off between objectives thoroughly so that decision making can be carried out after inspection of all possible optimal solutions. In Figure 5, the weights for several solutions on the Pareto front curve are displayed. Note that choosing a lower weight prioritizes the stability robustness, while a higher one promotes transparency. To obtain an optimal solution by the single criterion optimization (under scalarization) requires a proper choice for the weight, which is unknown a priori. Inspecting Pareto front curve reveals that such a weight cannot be determined by intuition alone, and a thorough analysis is required so that the designer can make an informed decision.

\section{E. Design Selection}

An optimal solution is selected from the Pareto front curve in three steps. Considering the pHRI scenarios discussed in Section III-A, we illustrate by examples how to i) impose possible constraints on the optimization objectives, ii) consider secondary design criteria, and iii) select an optimal design by deciding on an optimal solution on the Pareto front curve.

1) Constraints on the Optimization Objectives: Certain constraints may be imposed on the optimization cost functions to ensure some level of performance of a given pHRI task. These constraints need not to be assigned prior to optimization and can be determined after inspecting the Pareto front curve.

Let us consider the Pareto front curve presented in Figure 5. After inspecting the plot, the designer may choose to impose an upper bound of $C<11$ on the parasitic impedance, as marked by the horizontal dashed-line. This line intersects the Pareto front curve at a robustness margin of $\rho=0.35$. On the other hand, for another pHRI task, stability robustness may be more critical than the parasitic impedance, if the environment and/or human arm impedance are prone to significant change. For example, inspecting Figure 4 reveals that any controller

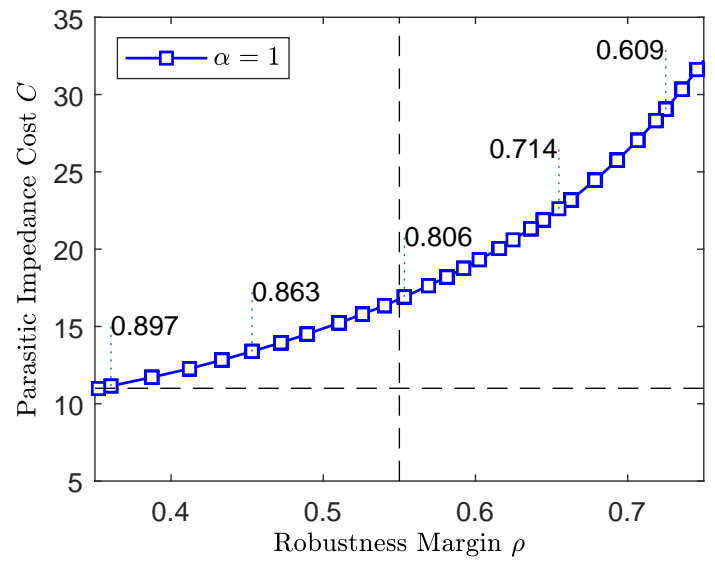

Fig. 5. Pareto front curve consisting of non-dominated solutions denoted by empty squares that are computed using multi-criteria optimization for IOAC $(\alpha=1)$. The vertical dotted-lines are for connecting the weights corresponding to sample solutions. The horizontal dashed-line denotes a sample upper bound for parasitic impedance at $C=11$. The vertical dashed-line represents the stability robustness of $\rho=0.55$. 


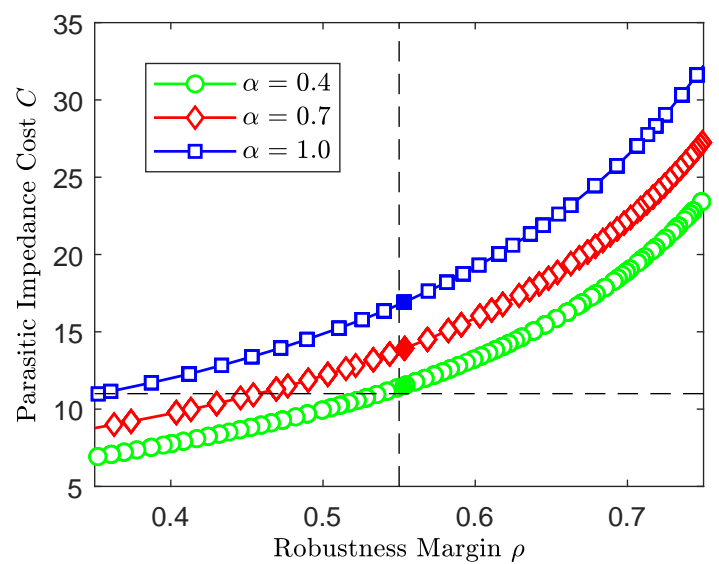

Fig. 6. Pareto front curves for IOAC $(\alpha=1)$, FOAC $(\alpha=0.4$ and 0.7$)$. The horizontal dashed-line denotes a sample upper bound of $C \leq 11$ for parasitic impedance. The vertical dashed-line represents a sample lower bound of $\rho \geq$ 0.55 for the stability robustness. The solutions denoted by filled markers are the optimal solutions selected, as detailed in Table II.

with a stability robustness margin of $\rho=0.55$ can maintain coupled stability up to $k_{\mathrm{eq}}=1610 \mathrm{~N} / \mathrm{m}$, the highest allowable stiffness in scenario S3. Prioritizing this stability margin, the designer can introduce the stability robustness margin of $\rho=0.55$ as a lower bound as depicted by the vertical dashed-line in Figure 5. This choice eliminates all the optimal solutions with $\rho<0.55$ from any further consideration.

Same constraints can also be applied to the optimal solutions on Pareto front curves of other integration orders, presented in Figure 6 . Note that, when both $C \leq 11$ and $\rho \geq 0.55$ constraints are simultaneously considered, there exits no optimal solution for IOAC, while there are such optimal solutions for FOAC with $\alpha=0.4$ and $\alpha=0.7$.

2) Considering Other Design Criteria: For the case study, stability robustness and transparency are chosen as the optimization objectives. However, once a thorough understanding of the trade-off between these objectives is achieved, the designer is free to introduce new design criteria to further narrow down the set of optimal solutions. As an example, noting that intended movements of human arm is bandlimited [53], we introduce an additional criterion for the pHRI task. In particular, we introduce a new criteria not captured by the optimization objectives and require the cut-off frequency of the closed-loop system to be larger than the bandwidth of the human arm's intended movements.

During the execution of a pHRI task, the user physically interacts with a robot and environment whose reflected impedance is characterized by $Z_{\text {disp }}(s)=F_{h}(s) / V(s)$, as in (3). To study the cut-off frequency of this closed-loop system, the transfer function $T_{\text {disp }}(s)=X(s) / F_{h}(s)$ is calculated using the reflected impedance and this cut-off frequency $\omega_{c}$ is computed for all the remaining solutions on the Pareto front. In Figure 7, these cut-off frequencies $\omega_{c}$ are presented as a function of stability robustness $\rho$.

3) Deciding on an Optimal Controller: As $k_{e}=610 \mathrm{~N} / \mathrm{m}$ is the lower bound on the environment stiffness for scenario $\mathrm{S} 3$, the cut-off frequencies $\omega_{c}$ computed for this stiffness level are considered for the design, noting that, an increase in the environment stiffness $k_{e}$ also increases $\omega_{c}$ (Figure 7). Given

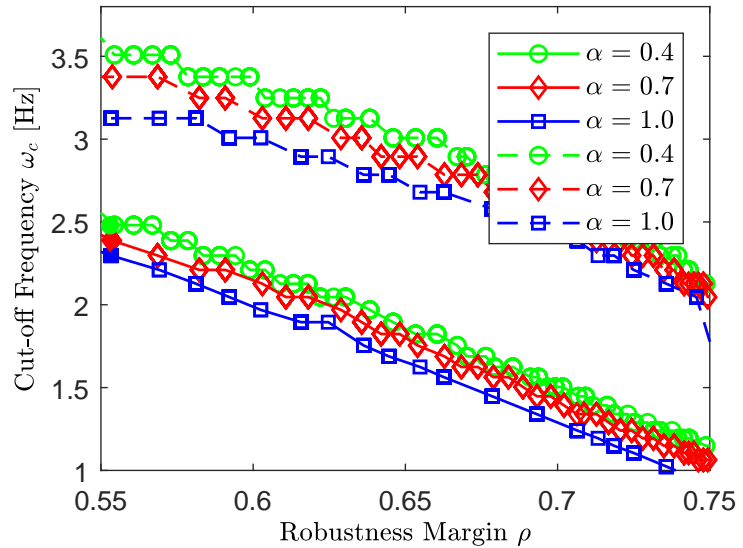

Fig. 7. The cut-off frequency $\omega_{c}$ of $T_{\text {disp }}(s)$ as a function of $\rho$ for the optimal solutions with $\rho \geq 0.55$. Solid and dashed lines are for $k_{e}=610$ and $k_{e}=$ $1010 \mathrm{~N} / \mathrm{m}$, respectively. The solutions represented by filled markers denote the optimal solutions selected, as detailed in Table II.

TABLE I

THE OPTIMAL CONTROLLER PARAMETERS, COST FUNCTION VALUES, AND OPTIMIZATION WEIGHTS FOR $\omega_{c} \geq 2.3 \mathrm{~Hz}$ AND $\rho \geq 0.55$.

\begin{tabular}{cccccc}
\hline$\alpha$ & $m_{F}\left[\mathrm{kgs}^{\alpha-1}\right]$ & $b_{F}[\mathrm{Ns} / \mathrm{m}]$ & $\rho$ & $C$ & $w$ \\
\hline 1 & 3.2 & 90 & 0.553 & 16.9 & 0.796 \\
0.7 & 6.0 & 74 & 0.568 & 14.5 & 0.755 \\
0.4 & 16.7 & 56 & 0.594 & 13.0 & 0.737 \\
\hline
\end{tabular}

that the bandwidth of the intended movements of human arm is around $2 \mathrm{~Hz}$ [14], [53], with a tolerance of $15 \%$, the solutions leading to $\omega_{c}<2.3 \mathrm{~Hz}$ are also eliminated from any further consideration.

An optimal solution that satisfies $\omega_{c} \geq 2.3$ was selected as the final design for each integration order (see Table I). We observe that FOAC (with $\alpha=0.4$ and $\alpha=0.7$ ) is a better choice than IOAC (with $\alpha=1$ ) since FOAC results in higher stability robustness $\rho$ and lower parasitic impedance $C$, leading to more robust and transparent design as presented in Table I.

An important advantage of Pareto methods is that designer only needs to decide on an optimal solution and the tradeoff between objectives after obtaining the Pareto front. The weighting parameter, $w$, for each sample design is given in Table I. Note that values of $w$ for each controller is different and these values cannot be easily determined by intuition a priori. A thorough knowledge on the trade-off characteristics is needed before deciding on these values.

\section{F. Comparison of Interaction Controllers}

Using Pareto optimization approach not only allows the designer to make an informed decision by inspecting all optimum solutions and studying the trade-off between the objectives, but also enables fair comparisons among various interaction controllers, possibly having different structures.

A close inspection of the Pareto front curves in Figure 6 reveals an interesting and important result: The Pareto front curve for the IOAC is completely dominated by those of the FOACs. In other words, parasitic impedance under IOAC is always larger than those of FOACs for the same robustness margin; hence, FOAC allows a better compromise between 
TABLE II

PARAMETERS AND THE PARASITIC IMPEDANCE $C$ OF THE OPTIMAL CONTROLLERS FOR SCENARIO S3.

\begin{tabular}{ccccc}
\hline$\alpha$ & $m_{F}\left[\mathrm{kgs}^{\alpha-1}\right]$ & $b_{F}[\mathrm{Ns} / \mathrm{m}]$ & $C$ & $w$ \\
\hline 1 & 3.2 & 90 & 16.9 & 0.796 \\
0.7 & 5.8 & 71 & 13.9 & 0.770 \\
0.4 & 15.4 & 49 & 11.5 & 0.776 \\
\hline
\end{tabular}

the stability robustness and transparency. Furthermore, it can be easily observed that the Pareto front curve for integration order $\alpha=0.4$ is superior to the others. Hence, the best compromise between stability robustness and transparency is obtained under $\alpha=0.4$ among the controllers considered in this study.

\section{A CONTROLlED PHRI EXPERIMENT: CONTINUOUS CONTACT WITH ENVIRONMENT}

The controllers tabulated in Table II were implemented for a pHRI experiment and a thorough comparison is reported in this section. In particular, optimal FOAC with integration orders of $\alpha=0.4$ and $\alpha=0.7$ that have the same stability robustness as the optimal IOAC $(\rho=0.553)$ were designed and implemented, to experimentally compare their transparency performance. The task involved contact interactions with a nonlinear environment formed by two linear springs that engage at different positions of the end-effector.

\section{A. Experimental Procedure}

In the experimental setup, human operator manipulates the robot to interact with an environment, where the environment stiffness is varied based on the depth of compression. In particular, two different springs are used as depicted in Figure 8.

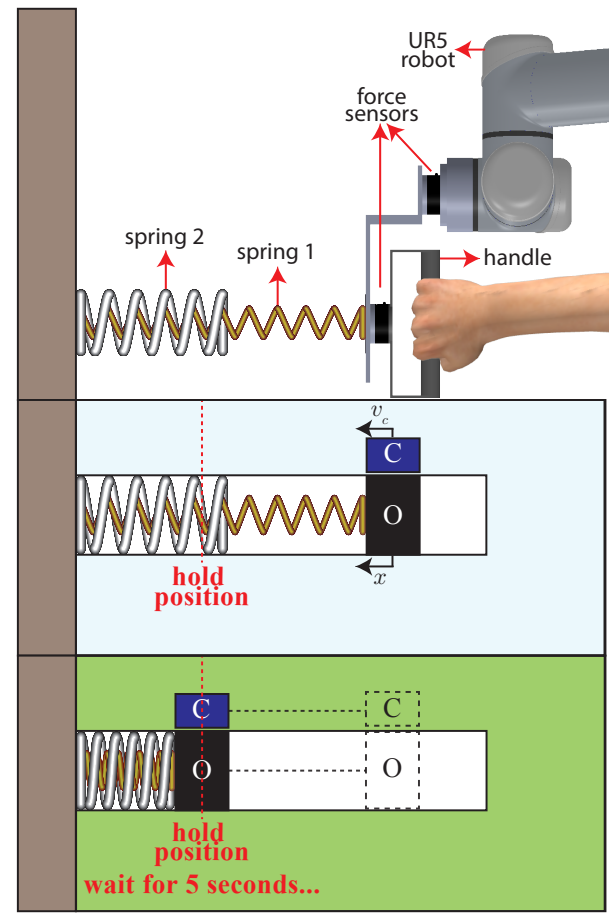

(a)

Fig. 8. The experimental setup (a) used, and the visual feedback (b,c) displayed to the subjects during the experiments.
Spring 1 with $k_{1}=610 \mathrm{~N} / \mathrm{m}$ is connected between a fixed support and the end-effector of a UR5 robot (Universal Robots Inc.), while Spring 2 with $k_{2}=400 \mathrm{~N} / \mathrm{m}$ becomes active when the depth of compression is larger than $45 \mathrm{~mm}$. Hence, the environment stiffness $\left(k_{e}\right)$ may change abruptly from $610 \mathrm{~N} / \mathrm{m}$ to $1010 \mathrm{~N} / \mathrm{m}$ during the interaction.

The subject grasps the handle to guide the end-effector and compresses the springs. The springs simply represent a layered environment in which the robot guided by a human operator is in continuous contact with, as in drilling a wall or inserting a needle into soft tissue. In the experiments, the subjects are asked to compress the springs up to a depth of $55 \mathrm{~mm}$, called as the ramp phase (Figure 8b), and then, hold it at that position for $5 \mathrm{~s}$, called as the hold phase (Figure 8c). In order to make the compression rate equal for each subject, a visual cursor (the dark blue rectangle, labelled as $\mathrm{C}$ in Figure $8 \mathrm{~b}$ ) moving with a constant speed $\left(v_{c}=20 \mathrm{~mm} / \mathrm{s}\right)$ is displayed on the computer screen. The other details of the experimental setup is the same as the one reported in [38].

Ten subjects ( 5 males and 5 females, average age $=29 \pm 6$ ) participated in this experiment. The subjects gave informed consent about their participation in the experiment. The experimental study was approved by the Ethical Committee for Human Participants of Koc University.

We compared subjects' performance for the controllers tabulated in Table II. Hence, three different controllers, IOAC $(\alpha=1)$, FOAC $(\alpha=0.7)$, and FOAC $(\alpha=0.4)$ were tested in this experiment. The task was repeated 10 times for each controller, resulting in $30(3 \times 10)$ trials in the experiment, which were displayed to the subjects in random order. The order was same for each subject. All trials were performed one after the other without any breaks. Prior to the experiment, each subject was given a training session of $15(3 \times 5)$ trials to get herself/himself familiar with the setup.

\section{B. Data Collection and Performance Metrics}

During the experiment, the robot constrains the motion of the subjects along a horizontal line while they compress the springs. The force $F_{h}$ applied by the subject is the sum of the forces required for compressing the springs $\left(F_{e}\right)$ and generating the motion trajectory of the robot (i.e. interaction force $\left.F_{\text {int }}\right) ; F_{h}=F_{e}+F_{\text {int }}$, where $F_{e}=k_{e} \Delta x$ ( $\Delta x$ is the amount of compression) is not influenced by the controller. $F_{\text {int }}$ is the interaction force measured by a force sensor (Mini40, ATI Inc.), filtered, and fed back to the admittance controller. The force applied by the subject $F_{h}$ (measured by a second sensor, Mini40, ATI Inc.) and the interaction force $F_{\text {int }}$ are linearly dependent on each other, since $F_{e}$ depends on the amount of compression only, and is independent of viscous and inertial effects coming from the controller and the robot. As the portion of the force applied by the subject to overcome the parasitic impedance of the robot is the interaction force, by inspecting this force alone, we can compare the transparency performance of the admittance controller for $\alpha=1, \alpha=0.7$, and $\alpha=0.4$.

We use average interaction force $\left(F_{\text {int }}^{\text {ave }}=1 /\left(t_{e}-\right.\right.$ $\left.\left.t_{b}\right) \int_{t_{b}}^{t_{e}}\left|F_{\text {int }}(t)\right| d t\right)$ to quantify the interaction performance un- 
der different controllers, and average force applied by human subjects $\left(F_{h}^{\text {ave }}=1 /\left(t_{e}-t_{b}\right) \int_{t_{b}}^{t_{e}}\left|F_{h}(t)\right| d t\right)$ to investigate the human effort, where, $t_{b}$ and $t_{e}$ are the beginning and ending times of a phase (ramp or hold), respectively. We also inspect the energy consumed to overcome the parasitic impedance of the robot $\left(E_{\text {int }}(t)=\int_{t_{i}}^{t} F_{\text {int }}(t) v(t) d t\right)$ as a function of time $t$, and then use it for estimating the total energy consumption $\left(E_{\text {int }}^{\text {tot }}=E_{\text {int }}\left(t_{f}\right)\right)$, where $t_{i}$ and $t_{f}$ are the beginning and ending times of a trial. Moreover, the total work done by the human $\left(W_{h}=\int_{t_{i}}^{t_{f}} F_{h}(t) v(t) d t\right)$ is also investigated. In addition, peak amplitude of oscillations in interaction force $A_{F_{\text {int }}}$, force applied by human $A_{F_{h}}$, and end-effector position $A_{P}$ are computed using fast Fourier transform (FFT) analysis as measures to compare the robustness under each controller.

\section{Data Analysis}

For each subject, the performance metrics $E_{\text {int }}^{\text {tot }}$ and $W_{h}$ were calculated for the entire duration, $F_{\text {int }}^{\text {ave }}$ and $F_{h}^{\text {ave }}$ were computed for the ramp phase, $A_{F_{\text {int }}}, A_{F_{h}}$, and $A_{P}$ were evaluated for the hold phase of all trials and then the mean values were normalized for the analysis (Figures 9, 10, and 11).

We performed one-way ANOVA to investigate the statistical significance of these results. In all statistical analyses, a significance level of $p=0.005$ was used for the null hypothesis.

\section{Results}

Figures $9 \mathrm{a}, 9 \mathrm{~b}$, and $9 \mathrm{c}$ present the average peak amplitude of oscillations in force applied by the subjects $A_{F_{h}}$, interaction force $A_{F_{\text {int }}}$, and end-effector position $A_{P}$ during the hold phase, respectively. Although these values were slightly lower for FOAC, the differences were not significant.

Figures 10a and 10b illustrate the average energy consumed to overcome the parasitic impedance of the robot $E_{\mathrm{int}}^{\mathrm{tot}}$, and the work done by the subjects $W_{h}$, respectively. We observed a statistically significant effect of controller on $E_{\text {int }}^{\text {tot }}$. Specifically, it was significantly lower under FOAC than that of IOAC. Moreover, it was the lowest under FOAC $(\alpha=0.4)$. In addition, FOAC slightly reduced the work done by the subjects.

Average interaction force $F_{\text {int }}^{\text {ave }}$, and force applied by the subjects $F_{h}^{\text {ave }}$ during the ramp phase were depicted in Figures 11a

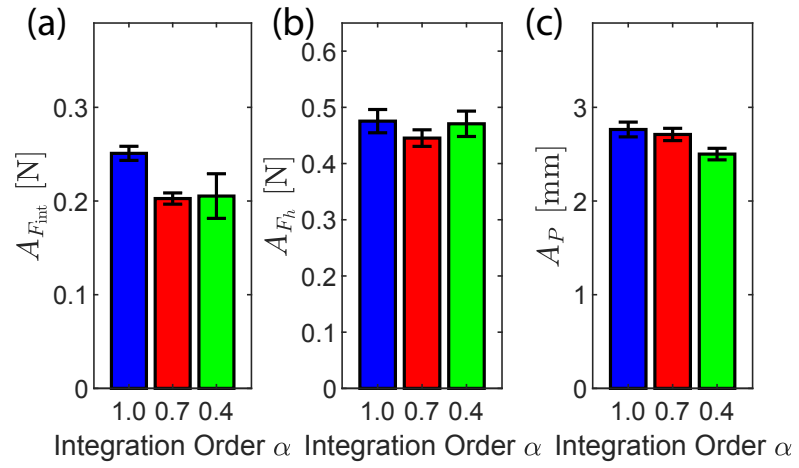

Fig. 9. The means and the standard errors of means of normalized performance metrics; peak amplitude of oscillations in (a) force applied by the subjects, $A_{F_{h}}$, (b) interaction force, $A_{F_{\text {int }}}$, and (c) end-effector position, $A_{P}$, during the hold phase.

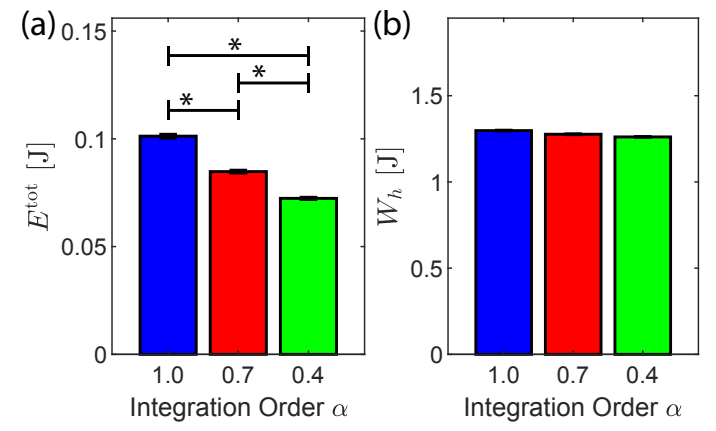

Fig. 10. The means and the standard errors of means of normalized performance metrics; (a) average energy consumed to overcome the parasitic impedance of the robot, $E_{\text {int }}^{\text {tot }}$, and (b) the work done by the subjects, $W_{h}$ (Horizontal bars with * on top indicate statistical significance between the results of the two corresponding controllers).

and $11 \mathrm{~b}$, respectively. We observed a statistically significant effect of controller on $F_{\text {int }}^{\text {ave }}$. Specifically, $F_{\text {int }}^{\text {ave }}$ was significantly lower under FOAC than that of IOAC. Moreover, $F_{\text {int }}^{\text {ave }}$ was the lowest under FOAC $(\alpha=0.4)$.

\section{E. Discussion}

We compared the performance of FOAC with that of IOAC for the pHRI task of compressing the two-stage spring system. Optimal admittance controllers with the same level of stability robustness were tested in our experiment and their transparency performances were compared.

None of the subjects experienced any instability while interacting with the robot during the experiments. The fact that no instability was observed provides evidence that each controller displayed sufficient level of stability robustness, as they were designed to tolerate even the highest equivalent stiffness during the task.

In order to compare the energy dissipation characteristics of these controllers, the amplitude of oscillations in force applied by the subjects, $A_{F_{h}}$, interaction force $A_{F_{\text {int }}}$, and endeffector position of the robot, $A_{P}$, were used. We did not expect a significant difference between these values during the hold phase (Figure 9), where the equivalent stiffness is the highest. Since each controller was designed to maintain the robust stability up to $k_{\text {eq }}=1610 \mathrm{~N} / \mathrm{m}$, their energy dissipation capacities should be similar.

We expected that the energy consumed to overcome the parasitic impedance of the robotic system during the ramp
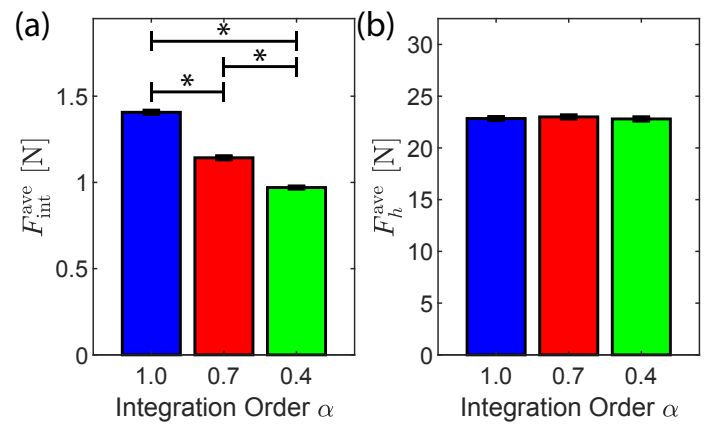

Fig. 11. The means and the standard errors of means of normalized performance metrics; (a) average interaction force, $F_{\text {int }}^{\text {ave }}$, and (b) force applied by the subjects, $F_{h}^{\text {ave }}$, during the ramp phase (Horizontal bars with $*$ on top indicate statistical significance between the results of the two corresponding controllers). 


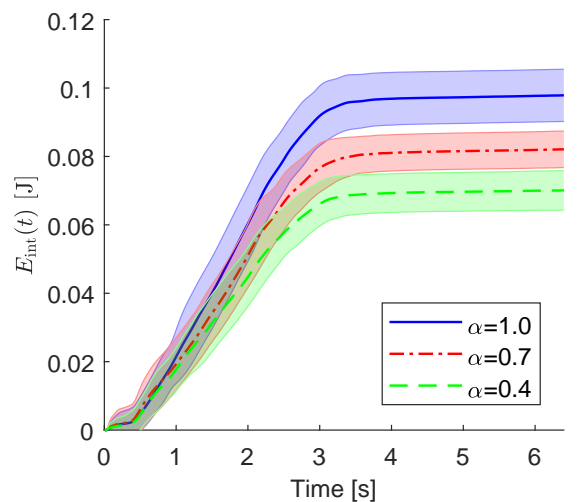

Fig. 12. Energy consumed to overcome the parasitic impedance of the robot as a function of time, $E_{\text {int }}(t)$.

phase would be significantly lower under FOAC for $\alpha=0.4$, as it was found to yield the lowest parasitic impedance in our computational analysis as presented in Table II. Indeed, the parasitic impedance was lower under FOAC in our experimental study as depicted in Figures 10a and 12. Furthermore, average interaction force during the ramp phase was reduced as the integration order was lowered, as can be observed in Figure 11a. The fact that both the energy consumption and the force to overcome the parasitic impedance were lower under FOAC revealed that a higher transparency was achieved with this controller. On the other hand, the difference between IOAC and FOAC in terms of the average force applied (Figure 11b) and the work done by the subjects (Figure 10b) was not significant during the ramp phase since compressing the springs required a significant portion of the total energy consumed by the subjects during the task.

\section{Discussion AND CONCLUSION}

In this study, we presented a multi-criteria optimization framework for synthesizing interaction controllers. In particular, we investigated the trade-off between transparency and stability robustness in pHRI systems and proposed a computational approach for optimal design of interaction controller.

Our computational approach resembles to the complementary stability analysis suggested in [25]. However, while their method relies on prioritization of one objective over another and imposing stability as a constraint, our approach advocates computation of the Pareto front that allows the designer to make an informed decision by studying all optimal solutions.

Pareto front solution not only can be used to select an optimal solution for the task at hand, but the same Pareto front may be utilized to select optimal controllers when the task changes, without a need for re-run of the analysis. For instance, in case the same pHRI system is to be used for the execution of another task where the environmental stiffness is higher than that of the previous task, the designer can select a new design on the Pareto front curve with a higher stability robustness to accommodate this change, as all possible optimal solutions are already available on the Pareto front.

The proposed design framework not only enables to optimize the controller parameters for the best trade-off performance, but also allows for interaction controllers with different structures to be compared rigorously. Note that, for interaction

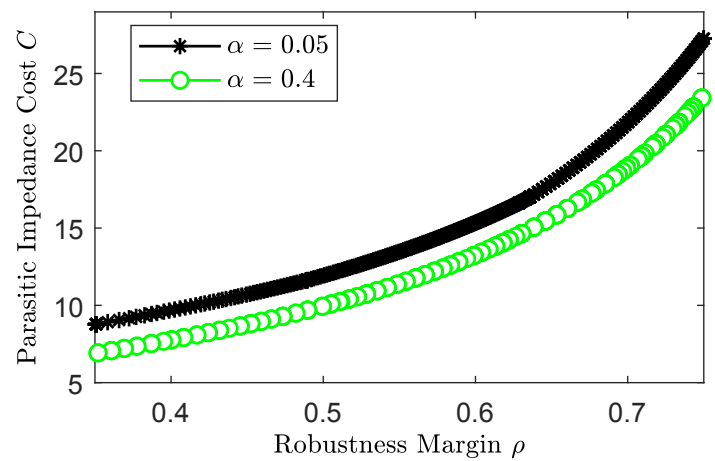

Fig. 13. Pareto front curves for FOAC with $\alpha=0.4$ and $\alpha=0.05$.

controllers having different forms or types than the ones considered in this study, Pareto front curves do not necessarily dominate each other, that is, some could be superior to others for a specific range of stability robustness while being inferior to the others for another range. In general, Pareto methods can precisely characterize which structure is superior for which range of objectives. Consequently, it can be emphasized that the Pareto front approach provides an objective comparison tool for interaction controllers of different forms and types, since it allows the designer to study, inspect, and compare all optimal controllers and trade-offs simultaneously.

We demonstrated the practical implementation of our approach for the design of integer and fractional order admittance controllers. A fair comparison between FOAC and IOAC is also presented, thanks to the Pareto front curves. Analysing the Pareto front curves suggested that the curve for IOAC is inferior to that of FOAC. Hence, FOAC allowed a better compromise between stability robustness and transparency. Moreover, the results of our computational analysis showed that, for the same robustness margin, $\alpha=0.4$ results in the lowest parasitic impedance allowing the highest transparency among the integration orders considered in this study.

To further study the effect of integration order, we computed the Pareto front curve of FOAC with $\alpha=0.05$. This Pareto front curve is presented in Figure 13 together with the Pareto front curve for FOAC with $\alpha=0.4$. It can be observed from Figure 13 that Pareto front curve of FOAC with $\alpha=0.4$ is also superior than that of FOAC with $\alpha=0.05$. These results indicate that the effect of integration order is not trivial and there exists an optimal integration order in terms of the objective functions considered. While $\alpha=0.4$ provides the best value among the integration orders considered, further analysis is required to compute the optimal order.

We also verified the computational design by an experimental pHRI task that involved contact interactions with a nonlinear environment formed by a two-stage spring. This onedimensional scenario was chosen for the following reasons: i) The computational design of the controllers had been conducted for a single-input single-output (SISO) LTI system; hence, the experiments should match this design for a rigorous verification. ii) It is easier to conduct, quantify and present human subject experiments with relatively simple tasks, as more complex tasks are likely to introduce confounding effects. iii) This scenario captures the sudden changes in environment parameters, emulating a worst-case interaction 
as the total stiffness of the human-robot-environment system has been shown to have the most dominant effect on the coupled stability. iv) This scenario represents a simplified version of many practical tasks involving contact interactions with various environments, such as needle insertion or drilling.

Needle insertion is a commonly used task in many medical procedures, including biopsies, brachytherapy, and epidural anesthesia. During a needle insertion, the needle is advanced into the soft tissue by maintaining a certain direction as the deviation of the needle in other directions can break the needle with undesired consequences. Along these lines, a needle insertion is mostly a one-dimensional task. During a needle insertion task, the operator advances the needle into the tissue by appreciating the magnitude of the interaction forces. A twostage spring system emulates the essence of this interaction as follows: The insertion of a needle into soft tissue starts with the deformation of the soft tissue (displaying a relatively stiff behaviour) and this is captured by the compression of Spring 1 in the experimental scenario. This deformation continues until rupture occurs, where a mixed stage of penetration and deformation starts. At this stage, as the needle continues its movement through the soft tissue, the forces tend to increase (displaying a different stiffness than the previous layer) until the needle reaches to another layer and this is captured by the activation of Spring 2 after a certain depth of compression.

To summarize, the experimental scenario in this study is considered as a representative simplification of a practical one-dimensional task. This choice enables experimental validation of the computational design, rigorous comparison of performance of the controllers considered and increased the digestibility of the procedure and the results. While it may be possible to test the proposed approach on other scenarios that require execution in multi-dimensions, such an extension will require extra effort to extend the proposed computational methodology. Such extensions are not considered as part of this study and will be left as a future research direction. Similarly, nonlinear or adaptive controllers are not considered in order to utilize powerful analysis tools available for LTI systems. We plan to investigate adaptive interaction controllers as part of our future work as suggested in [54].

\section{ACKNOWLEDGMENT}

The authors would like to thank to Prof. A. Kucukyilmaz for her valuable comments. Moreover, Y.A. thanks to A. Aytekin, O. Caldiran, E.S. Emgin, G. Serhat, and C. Yesil for fruitful discussions during the study. The Scientific and Technological Research Council of Turkey (TUBITAK) supported this work under contract EEEAG-117E645.

\section{REFERENCES}

[1] R. Ikeura, H. Monden, and H. Inooka, "Cooperative motion control of a robot and a human," in IEEE International Workshop on Robot and Human Communication, July 1994, pp. 112-117.

[2] A. Mörtl, M. Lawitzky, A. Kucukyilmaz, M. Sezgin, C. Basdogan, and S. Hirche, "The role of roles: physical cooperation between humans and robots," The International Journal of Robotics Research, vol. 31, no. 13, pp. 1656-1674, 2012.

[3] T. Wojtara, M. Uchihara, H. Murayama, S. Shimoda, S. Sakai, H. Fujimoto, and H. Kimura, "Human-robot collaboration in precise positioning of a three-dimensional object," Automatica, vol. 45, no. 2, pp. 333-342, 2009.
[4] M. Tavakoli, R. V. Patel, and M. Moallem, "A haptic interface for computer-integrated endoscopic surgery and training," Virtual Reality, vol. 9, no. 2, pp. 160-176, Mar 2006.

[5] A. U. Pehlivan, D. P. Losey, and M. K. O'Malley, "Minimal assist-asneeded controller for upper limb robotic rehabilitation," IEEE Transactions on Robotics, vol. 32, no. 1, pp. 113-124, Feb 2016.

[6] D. Reintsema, K. Landzettel, and G. Hirzinger, DLR's Advanced Telerobotic Concepts and Experiments for On-Orbit Servicing. Berlin, Heidelberg: Springer Berlin Heidelberg, 2007, pp. 323-345.

[7] M. A. Goodrich and A. C. Schultz, "Human-robot interaction: a survey," Foundations and Trends in Human-Computer Interaction, vol. 1, no. 3, pp. 203-275, Jan. 2007.

[8] A. D. Santis, B. Siciliano, A. D. Luca, and A. Bicchi, "An atlas of physical human-robot interaction," Mechanism and Machine Theory, vol. 43, no. 3, pp. 253-270, 2008.

[9] A. Ajoudani, A. M. Zanchettin, S. Ivaldi, A. Albu-Schäffer, K. Kosuge, and O. Khatib, "Progress and prospects of the human-robot collaboration," Autonomous Robots, vol. 42, no. 5, pp. 957-975, Jun 2018.

[10] D. P. Losey, C. G. McDonald, E. Battaglia, and M. K. O’Malley, "A review of intent detection, arbitration, and communication aspects of shared control for physical human-robot interaction," Applied Mechanics Reviews, vol. 70, no. 1, 02/2018 2018.

[11] T. Tsumugiwa, R. Yokogawa, and K. Yoshida, "Stability analysis for impedance control of robot for human-robot cooperative task system," in IEEE/RSJ International Conference on Intelligent Robots and Systems, 2004, pp. 3883-3888.

[12] V. Duchaine and C. M. Gosselin, "Investigation of human-robot interaction stability using lyapunov theory," in IEEE International Conference on Robotics and Automation, 2008, pp. 2189-2194.

[13] W. Gallagher, D. Gao, and J. Ueda, "Improved stability of haptic humanrobot interfaces using measurement of human arm stiffness," Advanced Robotics, vol. 28, no. 13, pp. 869-882, 2014.

[14] F. Dimeas and N. Aspragathos, "Online stability in human-robot cooperation with admittance control," IEEE Transactions on Haptics, vol. 9, no. 2, pp. 267-278, 2016

[15] Y. Aydin, O. Tokatli, V. Patoglu, and C. Basdogan, "Stable physical human-robot interaction using fractional order admittance control," IEEE Transactions on Haptics, vol. 11, no. 3, pp. 464-475, July 2018.

[16] F. E. Tosun and V. Patoglu, "Necessary and sufficient conditions for the passivity of impedance rendering with velocity-sourced series elastic actuation," IEEE Transactions on Robotics, vol. 36, no. 3, pp. 757-772, 2020 .

[17] J. E. Colgate and N. Hogan, "Robust control of dynamically interacting systems," Int. Journal of Control, vol. 48, no. 1, pp. 65-88, 1988.

[18] J. E. Colgate and G. G. Schenkel, "Passivity of a class of sampled-data systems: Application to haptic interfaces," Journal of Robotic Systems, vol. 14, no. 1, pp. 37-47, 1997.

[19] T. Hulin, A. Albu-Schffer, and G. Hirzinger, "Passivity and stability boundaries for haptic systems with time delay," IEEE Transactions on Control Systems Technology, vol. 22, no. 4, pp. 1297-1309, July 2014.

[20] B. Hannaford and J.-H. Ryu, "Time-domain passivity control of haptic interfaces," IEEE Transactions on Robotics and Automation, vol. 18, no. 1, pp. 1-10, Feb 2002.

[21] J.-H. Ryu, D.-S. Kwon, and B. Hannaford, "Stable teleoperation with time-domain passivity control," IEEE Transactions on Robotics and Automation, vol. 20, no. 2, pp. 365-373, April 2004.

[22] S. P. Buerger, H. I. Krebs, and N. Hogan, "Characterization and control of a screw-driven robot for neurorehabilitation," in IEEE International Conference on Control Applications, 2001, pp. 388-394.

[23] J. M. Dolan, M. B. Friedman, and M. L. Nagurka, "Dynamic and loaded impedance components in the maintenance of human arm posture," IEEE Transactions on Systems, Man, and Cybernetics, vol. 23, no. 3, pp. 698709, May 1993.

[24] T. Tsuji, P. G. Morasso, K. Goto, and K. Ito, "Human hand impedance characteristics during maintained posture," Biological Cybernetics, vol. 72, no. 6, pp. 475-485, 1995.

[25] S. P. Buerger and N. Hogan, "Complementary stability and loop shaping for improved human-robot interaction," IEEE Transactions on Robotics, vol. 23, no. 2, pp. 232-244, April 2007.

[26] A. Haddadi and K. Hashtrudi-Zaad, "Bounded-impedance absolute stability of bilateral teleoperation control systems," IEEE Transactions on Haptics, vol. 3, no. 1, pp. 15-27, 2010.

[27] D. A. Lawrence, "Stability and transparency in bilateral teleoperation," IEEE Transactions on Robotics and Automation, vol. 9, no. 5, pp. 624637 , Oct 1993. 
[28] K. Hashtrudi-Zaad and S. E. Salcudean, "Analysis of control architectures for teleoperation systems with impedance/admittance master and slave manipulators," The International Journal of Robotics Research, vol. 20, no. 6, pp. 419-445, 2001.

[29] P. G. Griffiths, R. B. Gillespie, and J. S. Freudenberg, "A fundamental tradeoff between performance and sensitivity within haptic rendering," IEEE Transactions on Robotics, vol. 24, no. 3, pp. 537-548, June 2008.

[30] I. Diaz, J. Gil, and T. Hulin, "Stability boundary and transparency for haptic rendering," in Advances in Haptics, M. H. Zadeh, Ed. INTECH, 2010, pp. 103-125.

[31] B. Willaert, D. Reynaerts, H. van Brussel, and E. B. V. Poorten, "Bilateral teleoperation: quantifying the requirements for and restrictions of ideal transparency," IEEE Transactions on Control Systems Technology, vol. 22, no. 1, pp. 387-395, Jan 2014.

[32] J. J. Abbott and A. M. Okamura, "Pseudo-admittance bilateral telemanipulation with guidance virtual fixtures," The International Journal of Robotics Research, vol. 26, no. 8, pp. 865-884, 2007.

[33] S. Hirche, A. Bauer, and M. Buss, "Transparency of haptic telepresence systems with constant time delay," in IEEE Conference on Control Applications, Aug 2005, pp. 328-333.

[34] T. Schau and A. Peer, "Parameter-space transparency analysis of teleoperation systems," in IEEE Haptics Symposium, March 2012, pp. 111116.

[35] J. E. Colgate and J. M. Brown, "Factors affecting the z-width of a haptic display," in IEEE International Conference on Robotics and Automation, 1994, pp. 3205-3210.

[36] D. W. Weir, J. E. Colgate, and M. A. Peshkin, "Measuring and increasing Z-width with active electrical damping," in Symposium on Haptic Interfaces for Virtual Environment and Teleoperator Systems, 2008, pp. 169-175.

[37] V. Chawda, O. Celik, and M. K. OMalley, "Evaluation of velocity estimation methods based on their effect on haptic device performance," IEEE/ASME Transactions on Mechatronics, vol. 23, no. 2, pp. 604-613, April 2018.

[38] Y. Aydin, O. Tokatli, V. Patoglu, and C. Basdogan, "Fractional order admittance control for physical human-robot interaction," in IEEE World Haptics Conference (WHC), June 2017, pp. 257-262.

[39] P. D. Labrecque and C. Gosselin, "Performance optimization of a multidof bilateral robot force amplification using complementary stability," in IEEE Conference on Control Applications, Sept 2015, pp. 519-526.

[40] S. Norouzzadeh, T. Lorenz, and S. Hirche, "Towards safe physical human-robot interaction: an online optimal control scheme," in IEEE International Symposium on Robot and Human Interactive Communication, Sept 2012, pp. 503-508.

[41] D. P. Losey and M. K. OMalley, "Trajectory deformations from physical humanrobot interaction," IEEE Transactions on Robotics, vol. 34, no. 1, pp. 126-138, Feb 2018

[42] C. Passenberg, R. Groten, A. Peer, and M. Buss, "Towards real-time haptic assistance adaptation optimizing task performance and human effort," in IEEE World Haptics Conference, June 2011, pp. 155-160.

[43] S. M. M. Rahman and R. Ikeura, "Weight-prediction-based predictive optimal position and force controls of a power assist robotic system for object manipulation," IEEE Transactions on Industrial Electronics, vol. 63, no. 9, pp. 5964-5975, Sept 2016.

[44] T. Hulin, "A practically linear relation between time delay and the optimal settling time of a haptic device," IEEE Robotics and Automation Letters, vol. 2, no. 3, pp. 1632-1639, July 2017.

[45] M. Gombolay, A. Bair, C. Huang, and J. Shah, "Computational design of mixed-initiative humanrobot teaming that considers human factors: situational awareness, workload, and workflow preferences," International Journal of Robotics Research, vol. 36, no. 5-7, pp. 597-617, 2017.

[46] M. Pearce, B. Mutlu, J. Shah, and R. Radwin, "Optimizing makespan and ergonomics in integrating collaborative robots into manufacturing processes," IEEE Transactions on Automation Science and Engineering, vol. 15 , no. 4, pp. 1772-1784, Oct 2018.

[47] P. D. Labrecque and C. Gosselin, "Variable admittance for phri: from intuitive unilateral interaction to optimal bilateral force amplification," Robotics and Computer-Integrated Manufacturing, vol. 52, pp. 1-8, 2018.

[48] P. Y. Papalambros and D. J. Wilde, Principles of Optimal Design: Modeling and Computation, 2nd ed. Cambridge University Press, 2000.

[49] R. T. Marler and J. S. Arora, "The weighted sum method for multiobjective optimization: new insights," Structural and Multidisciplinary Optimization, vol. 41, no. 6, pp. 853-862, Jun 2010.

[50] R. Unal, G. Kiziltas, and V. Patoglu, "A multi-criteria design optimization framework for haptic interfaces," in Symposium on Haptic Interfaces for Virtual Environment and Teleoperator Systems, March 2008, pp. 231-238.

[51] O. Tokatli and V. Patoglu, "Stability of haptic systems with fractional order controllers," in IEEE/RSJ International Conference on Intelligent Robots and Systems, Sept 2015, pp. 1172-1177.

[52] — Using Fractional Order Elements for Haptic Rendering. Cham: Springer International Publishing, 2018, pp. 373-388.

[53] T. L. Brooks, "Telerobotic response requirements," in IEEE International Conference on Systems, Man, and Cybernetics Conference, 1990, pp. 113-120.

[54] F. Ficuciello, L. Villani, and B. Siciliano, "Variable impedance control of redundant manipulators for intuitive humanrobot physical interaction," IEEE Transactions on Robotics, vol. 31, no. 4, pp. 850-863, 2015.

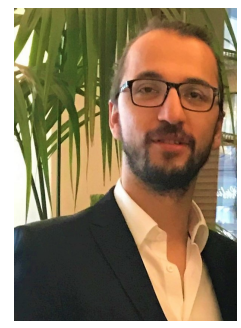

Yusuf Aydin Yusuf Aydin is currently a postdoctoral research fellow at Robotics and Mechatronics Laboratory at Koc University, Istanbul. He received his BSc dual degree in mechanical engineering and electrical and electronics engineering, MSc degree in mechanical engineering, and then, $\mathrm{PhD}$ degree in mechanical engineering from Koc University, Istanbul, in 2011, 2013, and 2019, respectively. He was awarded the prestigious TUBITAK BIDEB fellowship for his graduate studies. His research interests include physical human-robot interaction, haptics, robotics, control, optimization, and mechatronics.

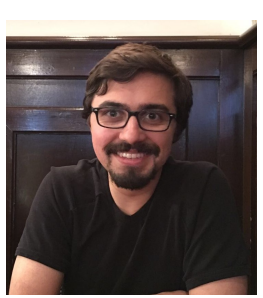

Ozan Tokatli Ozan Tokatli received his BSc, MSc and $\mathrm{PhD}$ degrees in Mechatronics Engineering from Sabanci University in 2008, 2010 and 2015, respectively. He worked as a post doctoral research associate in Haptics Laboratory at University of Reading. He is currently a research engineer in RACE, UKAEA which is a partner of the RAIN Hub. During his $\mathrm{PhD}$, he worked on haptic systems with fractional order controllers and during his postdoctoral research, he investigated the use of haptic interfaces for classroom use to enhance the learning. His current research interest is physical human robot interaction in hazardous environments. His research interests extend to design of haptic interfaces and control of robots for haptics and pHRI.

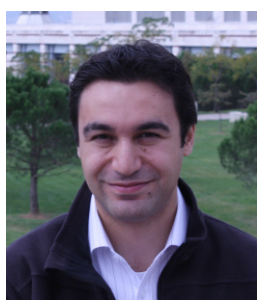

Volkan Patoglu Volkan Patoglu received his Ph.D. degree in Mechanical Engineering from the University of Michigan, Ann Arbor in 2005. He worked as a post doctoral research associate at Rice University. Currently, he is a professor at Sabanci University. His research is in the area of physical humanmachine interaction, in particular, design and control of force feedback robotic systems with applications to rehabilitation. His research extends to cognitive robotics. Dr. Patoglu has served as an associate editor for IEEE Transactions on Haptics (20132017) and is an associate editor for IEEE Transactions on Neural Systems and Rehabilitation Engineering and IEEE Robotics and Automation Letters.

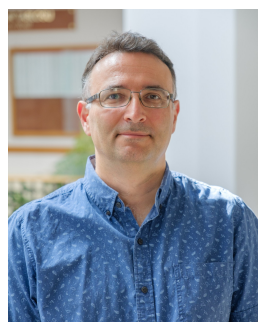

Cagatay Basdogan Cagatay Basdogan received the $\mathrm{PhD}$ degree in mechanical engineering from Southern Methodist University in 1994 . He is a faculty member in the mechanical engineering and computational sciences and engineering programs of Koc University, Istanbul, Turkey. He is also the director of the Robotics and Mechatronics Laboratory at Koc University. Before joining Koc University, he worked at NASA-JPL/Caltech, MIT, and Northwestern University Research Park. His research interests include haptic interfaces, robotics, mechatronics, biomechanics, medical simulation, computer graphics, and multi-modal virtual environments. He is currently the associate editor in chief of IEEE Transactions on Haptics and serves in the editorial boards of IEEE Transactions on Mechatronics, Presence: Teleoperators and Virtual Environments, and Computer Animation and Virtual Worlds journals. 